\title{
Adolescent Migrants and Reproductive Health in Kunming
}

\author{
Gu $\mathrm{He}^{1}$, , Tan qingli ${ }^{2}$ \\ 1 School of Public Management, Yunnan University of Economics and Finance, \\ Kunming, P.R.China, 650221 \\ 2 School of tourism and service trade, Yunnan University of Economics and Finance, \\ Kunming, P.R.China, 650221 \\ (Email:gutwo@ hotmail.com, tanqingli@ hotmail.com)
}

\begin{abstract}
As a marginal group, adolescent migrants' reproductive health status is little known. This paper mainly focus on individual and community factors effecting on adolescent migrants' reproductive health and needs. It is shown that two migrant groups should be more considered: unmarried migrants especially those living separately from their families and married men. Adolescent migrants face large obstacles for seeking services, and their access to information and services are very limited. Adolescent migrants live in certain community where social environment, social network and access to information and services affect their reproductive health status. Adolescent migrants just receive very limited and abridge information from their fellows and medias passively. Compared with other city residents, adolescent migrants are at disadvantages for economic, mental and culture side in process of seeking services.
\end{abstract}

Keywords: Adolescent migrant, Reproductive health.

\section{Introduction}

In China, reproductive health remains a new concept. In "The State Report of Population and Development of China" published by National Population and State Family Planning Commission of China on Sept.7th, 2004, the concept of reproductive health was quoted into the country population program in China, and relative practices was paid attention to. At present, the reproductive heath and AIDS prevention of the disadvantaged group especially that of migrant population, women and the poor, are remain very important and urgent challenges that China face. A just finished investigation conducting in several big cities where most migrant population of China gathers indicates that the situation of reproductive heath of the migrant population is worrying. An investigation of migrant workers' sex behaviors in Guang Zhou indicates that $50 \%$ of young female workers have had premarital sex experiences, and 30\% of male don't use any contraceptive methods (Zheng Lixing, 2003). Recently, an investigation of one thousand of female migrants aged from 15-24 in Shanghai indicates that nearly a half of female migrants admit that they ever had an unexpected pregnancy and the main reason is that they didn't have any contraceptive measures. It also indicates that a quarter of pregnant female migrants went to private clinics or took medicines by themselves to have an abortion (Gao Zhiguo, 2004). The information from relative hospitals indicates that among the patients who suffer from sex problems, more than $80 \%$ are migrant population, and $70 \%$ are male (GDPIC, 2003).

HIV/AIDS in China has been spread fast since the middle of 90th year in 20th century. In 2003, an investigation of the situation of HIV/AIDS in China was taken all over the country. The advance analysis result indicates that there are about 840 thousand HIV infected people in China and there are about 80 thousand AIDS patients among them. At present, the situation of HIV/AIDS in China is facing a danger that HIV/AIDS spreads from vulnerable group to common people in a large area. Migrant population is the vulnerable group which is most possible to be infected 
and spread the illness. So to find out the condition of reproductive health of migrant population and to supply them relevant social medical services is very important to improve reproductive health of migrant populations and prevent the spread of HIV/AIDS.

Being in southwest and bordering three countries which are Laos, Vietnam and Myanmar, Yunnan province is an undeveloped district in China. Economy in Yunnan is comparatively backward. The first HIV infected person in Yunnan was discovered in 1989. And in 2010, the provincial report announced that the number of HIV infected people and patient was 82,000 . Relevant experts estimated that the real number was 700 thousand or so and was the highest in China. Preventing HIV/AIDS has been an important work for the provincial government. One tenth of the whole population of Yunnan is gathered in Kunming city. According to the data of the sixth population census in 2010, the total resident population of Kunming city is $6,432,212$, and the migrant population is 1.98 million. The migrant population especially adolescent migrant population is the key point of preventing HIV/AIDS. To understand the condition of their reproductive health has important effect on improving relative policies formulation and carrying out relative services. In this research, two aspects are concentrated on to analysis the condition and relative needs of the reproductive health of migrant adolescents: personal factors and community factors.

\section{Adolescent Migrants and Reproductive Health in Kunming}

\subsection{Methodology}

Data on adolescent migrants and reproductive health structure are acquired from China's Research Plan for regional Research Initiative on Adolescent Migrants and reproductive Health in The Greater Mekong Region. Qualitative data were collected from three communities where many migrants live in. They are Fude, Xiqiao, Xiba communities. The amount of the sample of adolescent migrants is 79 , including 40 males and 39 females. There are also 13 doctors and nurses who serve reproductive health services and are mainly in public hospital, the community service centres of family planning and private clinic.

Our relevant information research concentrates on the investigations of reproductive health of migrants conducted around the country in recent years, as well as relevant policies of the different level government in China. The relative investigations and dissertations from research institutes as well as the communiqués from the government become our main information sources.

\subsection{Adolescent migrants in Kunming}

The migrant population of Kunming has been increasing since 1990. According to the data from the sixth census in 2010, the migrant population in Kunming is 1.98 million. The migrants mainly scatter in Wuhua, Panlong, Guandu and Xishan districts especially in Guandu and Xishan districts which border on the outskirts of the city and in which the migrant population occupies $48.84 \%$ and $17.9 \%$ of the whole district population respectively. Since the industries and services in Kunming are undeveloped, the ability of containing labors is limited. In fact, the jobs that migrants take are mainly hard work like building and carrying. The majority of migrants are from poor rural areas. $49 \%$ of them are from Yunnan; 34\% and 9\% of them are from Sichuan and Guizhou provinces respectively which have border with Yunnan; $8 \%$ of them are from other provinces in China.

\subsection{Individual factors}

\subsubsection{Gender}

Both the male and female adolescent migrants face the problems of reproductive health, but their concepts, attitudes and actions of reproductive health are very different. The differences may be rooted in physical condition, status and responsibility decided by social culture.

In the traditional chinese culture especially in rural areas, the male are thought more important than the female. Regarded as the mainstays of families and the masters of houses, the male own greater power than the female who mainly assistant and abbey the male as well as manage housework. Male adolescents usually think about reproductive health problems under the actual condition of their life while female adolescents pay more 
attention to the changes of the relationship of the female and the male caused by these problems as well as the physical consequences. The female responsibilities of these problems are emphasized by both the female and the male. Comparatively, the male responsibilities are not paid equal attention to and a number of interviewees agree that the male have more rights. What is interesting is, a number of male interviewees think the male have great responsibility for preventing from spreading STDs and HIV/AIDS. Apparently, the male will pay more attention to their duties when they realize sex may cause reproductive health problems on them. For unexpected pregnancies, female responsibility is emphasized more by both the male and the female.

\subsubsection{Marriage}

Marriage is an important factor influencing migrants' reproductive health. It has important influence on the condition of reproductive health of migrant adolescents especially on their acts of reproductive health. Traditionally, there is a large number of married people migrating with family in China. Besides, because of the severe family planning policy in China, the relative work about married adolescents especially the female migrants is a focal point for relative government apartments. The work also influences their acts of reproductive health directly.

Generally speaking, sex is restrained by marriage. However, it is just because of the un-equality of the two sexes on sex that married men are restrained less by marriage than married women being done. At a certain degree, marriage strengthens the un-equality of the two sexes on reproductive health. To bear babies is still quite important in marriage in China and especially in rural areas. To bear boys is the hope of married adolescents. Because of the responsibility for bearing and raising children, as well as the influence of family planning policy and traditional culture, married female adolescents' reproductive behaviors and sex are much more restrained than that of married male adolescents. Married men have absolute authority on sex with their wives, and they also have much more rights on sex beyond marriage. Although the restraints of marriage on male sex can't be denied, marriage actually maintains and strengthens the male authorities and rights on sex at a certain degree. Consciously or unconsciously, the female also become the guards maintaining male authorities and rights on sex. Male sex is tacitly approved at many times. Of course, this means they will face much more risks of involving reproductive health problems.

\subsubsection{Familial and Fellows Roles}

The factors of family and fellows influence migrant motivations, the choices of migrant places, living conditions, knowledge and acts of reproductive health of migrant adolescents directly or indirectly. In the traditional culture of china, family is very important, and family in rural areas is more important in people's mind than that in cities. In the rural areas of china, a majority of youth end their education after graduation from junior secondary school for personal reasons or economic reasons. Most of them were forced to go out to look for jobs because of limited land and limited sources of income in their hometowns. Because of lack of information in rural areas, it is family members or persons from the same village that become the main sources of information for young people who want to look for jobs outside, and that influence their choices of migrant places. Besides, the change of relationship of family members, such as parents divorce, re-marriage after divorce, sickness and death, is also an important reason of adolescents' migration.

For lack of information and backward education in rural areas, most adolescents lack knowledge about reproductive health. Family members and friends are the main sources of their limited knowledge of reproductive health. Comparatively, young adolescents especially female adolescents who live with family members are influenced greatly by family. Female adolescents' initial knowledge about catamenia come from their mothers or elder family members. When it comes to sex before marriage and cohabiting, they pay more attention to the family reaction. Elder adolescents living without family members are influenced greatly by friends. In traditional culture of china, sex is a forbidden topic in family. Both sex before marriage and cohabiting are not permitted. But in the fellows group which is made up with friends with similar ages and similar backgrounds, it won't meet any obstacles to talk about these topics. Migrant adolescents' opinions about sex before marriage and cohabiting are usually influenced by their friends.

2.3.4 Knowledge about reproductive health 
and reproductive health problems

Majority of adolescent migrants lack the basic knowledge about reproductive health. Actually, there are no formal educations on knowledge about RH in China, and students get quite few knowledge about $\mathrm{RH}$ from school. In rural areas, many adolescent finish their education after they graduate from secondary school or some leave school early, so these adolescents get very limited knowledge about $\mathrm{RH}$ from education system. On the other hand, their family members also seldom provide them knowledge about RH because of the influence of traditional culture and close social environment in rural areas. When these adolescents move to the city, they face a new environment. In here, they can get some knowledge about RH directly or indirectly, but most of knowledge is abridged. In general, adolescent migrant have few knowledge about $\mathrm{RH}$.

\subsection{Community factors}

When adolescent migrants move to Kunming, they have to live in a different community with their hometown. In this community, their lives are affected by the new social environment, social culture and custom, and social network, and their reproductive health status also are affected by these factors. At the same time, the access to information and services, and qualify of services also influence adolescent migrants status in the new community.

\subsubsection{Social environment}

Majority of migrants live in these areas between downtown and suburban, and the characters of these areas are typically different with other areas. Just like other cities in China, there is a great change for Kunming in recent years. The new city districts extend consistently, while a large of agricultural lands are occupied. However, some new districts are neglected because of the fault of city planning, and there are mass private buildings, and lack of the relevant managements and services in these areas with poor living facilities, and bad sanitations. Because of the household registration system, migrants are confined for jobs, lives and other sides. Most of them come from the poor rural areas, and their economic levels are very low. So most of them choose to live in these areas with lower living cost when they move to Kunming. These characters just are displayed in our study areas. Actually, these areas are isolated with other areas, to some extend, migrant live a special area or community where is quite different with other areas. There are many social problems in these areas, and the frequency of drug, prostitution, rob and other illegal crimes are very high in there. Majority of adolescent migrants think they lack security feeling in the community where they live in. Under that disordered environment, adolescent migrants are vulnerable to lose control of themselves or suffer from all kinds of problems, include of reproductive health problems.

\subsubsection{Social culture and social network}

There is a great difference for culture between urban area and rural areas. People have conservative views about sex because of traditional culture and poor information accesses in rural areas, especially poverty areas. City residents have more opening views about sex compared with those living in rural areas. In cities, people have more information accesses and are affected by diverse culture, so they have more chances to choose their life style. When adolescent migrate to city, they also are affected by city culture and custom. Majority of adolescent migrant can accept premarital sex and cohabit behavior and regard them as common behavior. On the other hand, they also think that premarital sex behavior are prohibited and regard as immorality behavior by many rural people. Actually, adolescent migrant are affected by two different cultures and custom, sometimes they express conflicting and inconsistent views about sex behaviors.

There is also a great difference for social network between adolescent migrants' living community and hometown. Compared with city, social relationship is more closed in rural area. In chinese rural area, social network based on kinship has very strong power. Rural people keep very closed relationship with their family and relatives in their lives and other social activities. Adolescents' behavior are supervised by their family and older relatives. To some extend, their sex attitude and behavior are restricted by this social network. When they move to city, this relationship becomes weaken. In new community, they develop new social network, and they get much influence from their fellows and others in their lives and social activities. The new relationship is more open, and lack of restricted power. So all kinds of views about sex and actual sex behavior are easy to 
accepted and tolerated by the community. Even to those adolescent migrants living with their family member, they also have much freedom about sex in here compared with their hometown.

2.4.3. Access to information and services

Compared with hometown, adolescent migrants have much access to information in the new community. Majority of them have few information about reproductive health before their moving, and they get much information in the city. Adolescent migrants get information from all kinds of newspapers, magazines, television and so on. Some also can get it from drug shop, clinic and hospital, and there is lack of these access to information in their hometown. Although they can get much information about reproductive health in city, these information are abridge on some extend. Actually, adolescent migrant still lack the basic knowledge about the reproductive health, especially the reproductive diseases and the preventative methods. Adolescent migrant can get some information from health facility, however, which mostly happens while they seek medical services, and these health facilities seldom provide information to them voluntarily.

In general, there are three kinds of health facilities which provide reproductive services in Kunming: public hospital, private hospital or clinic and different level of family planning centers. Many public hospitals locate in the center of the city and mainly provide service to city residents who can get much benefit from medical insurance. Private hospital or clinics have developed over past 10 years, many of them locate in those areas between downtown and suburban where a large number of migrants live in, and their customers mainly are migrants. In general, there are family planning centers which are supported by government in majority of communities, and they provide contraception information and service to married women. Adolescent migrants choose the private clinic which charge not too high when they meet all kinds of reproductive problems. It is mostly because of their limited economic ability. However, majority of private clinics lack eligible service provider and good medical instruments, and adolescent migrants have to put themselves at risk when they come to these. On the other hand, adolescent migrants have to pay more for medical cost when they come to public hospital to seek service because that majority of them come from the rural areas, and they are not covered by medical insurance. At the same time, the more complicated procedures also constrain adolescent migrant to choose public hospitals. Because of implement of family planning, chinese government establish the different level of family planning centers which provide all kinds of knowledge and service about contraception. However, according to the management rules about family planning for migrants made by State Department in 1998, unmarried adolescent migrant and married male migrant all are excluded to seek reproductive service from family planning center. Actually, in many health service providers' views, it is very important to provide knowledge about reproductive health to adolescent migrant. However, the related access to information and service are still very limited from the health facilities.

\subsubsection{Quality of services}

Although majority of adolescent migrants think that there are better medical instruments and quality of services in Kunming compared with hometown, they also complain about the higher medical cost and more complicated procedures. Usually, when adolescent meet reproductive problems, their first choice is to buy drug or let it be, and they have to go to hospital or clinic only if these problems become very serious. When they choose the kind of medical facility, their first choice is to go to the private clinic. Only if these problems or diseases can not be cured in private clinics, they go to public hospital. Which is related with adolescent migrants' economic status and mentality. In many adolescent migrants' views, one important problem is related with economy when they meet reproductive health problem. They have limited ability to pay service cost because of lower income. At the same time, they have to pay more for medical cost than city residents because that they are not covered by medical insurance. On the other hand, as a marginal group, adolescent migrants have to face all kinds of cultural and mental barriers while they seek service. Majority of them think that they are blamed by other people, and get much mental burden when they get reproductive problems. Under that burden, they have mental constrains to seek services and feel embarrassed to go to see a doctor or do not 
seek service in time, which causes their disease more serious.

On the side of health facility, public hospitals have enough ability to provide the better services because of their eligible service provider and good medical instruments compared with private hospitals or clinics. However, these hospitals can not provide the appropriate services to adolescent migrants with lower economic income because of lack of the related policies, and their large scale and complex management. In addition, many public facility locate in the center of the city, and it is difficult to serve those suburban communities where migrant live in to them. Traditionally, health facilities belong to the nation in China and private facilities have just developed in past 10 years. Majority of private facilities are private clinics which cause many problems because of lack of managements, and their poor medical instrument and unqualified service providers. It is very common that they make the wrong and omitted diagnosis or bring sequela to patients. On the other hand, majority of these clinics locate in those communities where migrant live in, and they do not require register and charge lower cost compared with public hospital, so many adolescent migrants choose them as the first choice even they know the relate risk.

\section{Conclusion}

As a marginal group, adolescent migrants' reproductive health status is little known. Our study mainly focus on individual and community factors effecting on adolescent migrants' reproductive health and needs. To adolescent migrant individuals, different migrants face different reproductive problems and risk. Through our investigation and analysis, two migrant groups should be more considered: unmarried migrants especially those living separately from their families and married men. Compared with male, female face much risk of physical and cultural influences caused by reproductive problems, however, they are obligated to take much duty. In addition, this group lacks the knowledge about reproductive health highly. They do not know basic contraception methods, and have limited access to reproductive information. When they face reproductive problems, they have to face mental and economic constrains in process of seeking services, and there is also lack of appropriate and effective services provided by health facilities for them. Married male is a special group among adolescent migrants, and they often are neglected in reproductive health researches. Compared with married females, married males are put in a higher status by social value and culture, and their sex rights are much stressed and accepted, which also make them face more risks of reproductive problems. Investigation shows that there is higher probability of making risk behaviors for married males. Information about reproductive health should be transmitted to them and their spouses, especially knowledge about risk behaviors causing reproductive diseases. Although these two groups should be focused, other migrants' reproductive health status and needs also can not be neglected. It also should be mentioned unmarried males' need for knowledge about reproductive health and married females' need for reproductive services. On the other hand, as a whole group, adolescent migrants face large obstacles for seeking services, and their access to information and services are very limited. Actually, individual factors are just one factor affecting adolescent migrants' reproductive health status. Another factor is related with community. Adolescent migrants live in certain community where social environment, social network and access to information and services affect their reproductive health status, and the effect from the latter is very direct. Mostly, adolescent migrants just receive very limited and abridge information from their fellows and medias passively. Compared with other city residents, adolescent migrants are at disadvantages for economic, mental and culture side in process of seeking services. Firstly, they have to face the obstacle from medical system. They lack medical insurance, and have to pay more for medical cost. And bureaucratic management also constrain adolescent migrant to choose public facilities. Because of policy constrain, limited human and material resource, family planning center just provide services to some migrants, practically married females at reproductive period. Mostly, private clinic become the first choice for adolescent migrant when they seek services. However, the quality of services provide by these clinics is very lower because of lack of managements, and their poor medical instrument and unqualified service 
providers, and adolescent migrant face more risk in this process.

\section{References}

[1] GDPIC. The study of migrant workers' reproductive health in Guangzhou. 2003.(In Chinese)

[2] Institute for Population, Fudan University. Reproductive health services and migrants' perception about reproductive health, reproductive behaviors and status in Shanghai. 2003.(In Chinese)

[3] Li Qiao-Chun, A report of reproductive health of women migrant workers in Guangdong Province, China. 2004.(In Chinese)
[4] The State Population and Family Planning Commission. The State Report of Population and Development of China. July. 2004. (In Chinese)

[5] The State Population and Family Planning Commission, The 2002 year report about family planning. 2002. (In Chinese)

[6] The State Population and Family Planning Commission, The tran-province migrants' marriage, bearing and contraception. 2001. (In Chinese) 